\title{
Clinical Considerations in Lung Cancer
}

Pallav Shah

Royal Brompton Hospital, London, UK

\section{Introduction}

Lung cancer remains one of the commonest malignancies, accounting for $20 \%$ of all cancers in men with a lifetime risk of 1 in 13 and $12 \%$ of all cancers in women with a lifetime risk of 1 in 23 [1]. In the United Kingdom roughly 40,000 new cases are recorded each year. The estimated incidence for lung cancer in males in 2005 in the United States was 92,305 with approximately 91,537 males expected to die from the disease [2]. The risk of lung cancer is about fourfold greater in men than in women and this increases with age: in the European Union the incidence of lung cancer is 7 per 100,000 for men and 3 per 100,000 for women at the age of 35 years, but in patients aged over 75 , the rates are 440 and 72 in men and women respectively [3]. Wide geographical variations in the incidence of lung cancer are also reported and this is primarily related to worldwide variations in smoking behaviour.

\section{Aetiology}

Smoking cigarettes is far and away the dominant risk factor in patients with lung cancer, accounting for $90 \%$ of lung cancers in men and almost $80 \%$ of cases in women. The relationship between smoking and lung cancer mortality was first established by Doll and Hill [4]. In their study, newly admitted patients with suspected lung, liver or bowel cancers were questioned. The results demonstrated conclusively that patients with a final diagnosis of lung cancer were more likely to be smokers than those without a final diagnosis of lung cancer. The critical study was a prospective study from a cohort of doctors on the medical register who were recruited via a letter in the British Medical Journal; there were 40,000 respondents. Over the subsequent two and half years there were 789 patient deaths, 36 of whom had lung cancer. Doll and Hill found a significant increase in the risk of lung cancer 
Cambridge University Press

978-0-521-87202-7 - Lung Cancer

Edited by Sujal R. Desai

Excerpt

More information

with tobacco consumption [5]. A recent update has also published the 50-year results of this landmark investigation [6].

Occupational asbestos exposure has also been shown to increase the risk of lung cancer, particularly with amphibolic froms of asbestos [7]. The effect is significantly increased in individuals who smoke (up to 16-fold) [8]. Radon, which is a decay product of uranium 238 and radon 226, can also accumulate in homes and some studies have demonstrated an increased risk of lung cancer [9]. Exposure to the following carcinogens has also been associated with an increased risk from lung cancer: arsenic, beryllium, bis-choromethyl ether, cadmium, chromium, nickel, polycyclic aromatic hydrocarbons and vinyl chloride. Consequently, a greater incidence of lung cancer has been observed in industries such as coal-gas, metal refining and smelting processes.

\section{Natural History}

Assuming the hypothesis that lung cancer grows from a single cell, it usually takes approximately 40 volume-doublings for the tumour to reach a diameter of $10 \mathrm{~cm}$, which is the average size of the tumour at death (Table 1.1) [10]. The average size at which tumours are diagnosed is $3 \mathrm{~cm}$ and they have usually undergone 33 volume doublings. Small cell cancers are the most rapidly dividing cancers and double in volume approximately every 29 days. Thus, small cell cancers have been present, on average, for about 2 years 4 months before becoming detectable. In contrast, adenocarcinomas of the lung are slow growing, doubling in volume every 161 days. Squamous cell carcinomas and poorly differentiated carcinomas tend to be somewhere in between, doubling in volume every 88 days.

Table 1.1. Natural history of untreated lung cancer (adapted from Geddes, 1979 [10])

\begin{tabular}{lcccc}
\hline & \multirow{2}{*}{$\begin{array}{c}\text { Volume doubling } \\
\text { time (days) }\end{array}$} & \multicolumn{3}{l}{ Time from malignant change (in years) } \\
\cline { 3 - 5 } Cell type & Earliest diagnosis & Usual diagnosis & death \\
\hline \multirow{3}{*}{ Small cell } & 29 & $1 \mathrm{~cm}$ & $3 \mathrm{~cm}$ & $10 \mathrm{~cm}$ \\
Poorly differentiated & 86 & 2.4 & 2.8 & 3.2 \\
Squamous & 88 & 7.1 & 8.2 & 9.4 \\
Adenocarcinoma & 161 & 7.1 & 8.4 & 9.6 \\
\hline
\end{tabular}


The growth rate of lung cancer also illustrates (Table 1.1) that the disease is usually diagnosed late in its natural history. Against this, it is noteworthy that most tumours are capable of metastasizing after about 20 volume doublings but not detectable until after 30 volume doublings. Hence the majority of patients have advanced disease by the time of presentation.

\section{Clinical Features}

\section{Symptoms and Signs due to Local Disease}

The majority of patients who are diagnosed with lung cancer have symptoms at presentation [11]. The most common symptoms are chronic cough with or without sputum production (Table 1.2). Excessive sputum production is an occasional feature of bronchoalveolar cell carcinoma. Haemoptysis is a symptom that frequently prompts patients to seek medical attention and is a presenting feature in up to $50 \%$ of cases. Chest pain is a common feature and may vary from dull vague pain on the side of the tumour or more severe pain due to chest wall or mediastinal invasion. Local invasion of adjacent structures such as ribs and vertebral bodies by the tumour may also cause severe persistent pain.

Table 1.2. Clinical features of lung cancer

\begin{tabular}{ll}
\hline Local disease & Intra-thoracic spread \\
\hline Symptoms & Symptoms \\
Cough & Chest wall pain \\
Productive sputum & Shoulder tip pain \\
Haemoptysis & Weakness in the hand \\
Chest pain & Hoarse voice \\
Weight loss & Headaches \\
& Facial swelling \\
Clinical signs & \\
Clubbing of finger nails & Clinical signs \\
Monophonic wheeze & Dilated neck veins \\
Focal wheezing & Facial plethora \\
Stridor & Horner's syndrome \\
& Wasting of the small muscles \\
& of the hand \\
\hline
\end{tabular}


Cambridge University Press

978-0-521-87202-7 - Lung Cancer

Edited by Sujal R. Desai

Excerpt

More information

Recurrent focal pneumonia and segmental pneumonia should raise the possibility of an obstructive lesion in the airways and should prompt further investigation. Unilateral and monophonic wheezes are less common features of an obstructive bronchial tumour. Stridor may occur where there is tracheal involvement.

\section{Symptoms and Signs due to Intra-thoracic Extension}

Extension of lung cancer to adjacent structures may also lead to clinical symptoms and signs. Breathlessness and chest pain may be caused by pleural involvement or pericardial involvement. The subsequent pleural or pericardial effusions may cause breathlessness, and in the case of pericardial involvement may also lead to cardiovascular compromise.

Right upper lobe tumours or adjacent mediastinal nodes may invade or externally compress the superior vena cava (SVC). Such patients then present with a relatively classical SVC syndrome comprising headaches, facial fullness or plethora and oedema with congested neck and chest veins. The SVC syndrome is a presenting feature in about $10 \%$ of patients with small cell lung cancer. Apical tumours may also extend to involve the superior sympathetic chain leading to a Horner's syndrome, and brachial plexus involvement causes shoulder and neck pain with atrophy of the small muscles of the hand. Left-sided tumours may compress the recurrent laryngeal nerve as it courses above the aortic arch leading to a hoarse voice and left vocal cord paralysis. Direct tumour invasion or enlarged mediastinal nodes may cause oesophageal compression and hence dysphagia.

\section{Symptoms and Signs due to Distant Spread}

Weight loss is a relative common complaint in patients with lung cancer, which is usually associated with a poor outcome; indeed a decrease of weight exceeding $20 \%$ of baseline body weight, in the preceding month, is often indicative of metastatic disease. Patients with liver metastases often present with weight loss. Lung cancer also frequently spreads to the adrenal glands, bone, brain and skin. Involvement of these sites may cause localized pain. Bone metastases can occur at any site but tend to occur in the ribs, vertebrae, humeral and femoral bones. With brain metastases there may also be neurological symptoms, such as confusion, personality changes and epileptic seizures. Supraclavicular and anterior cervical lymph nodes may be involved in up to $25 \%$ of patients and should be routinely assessed in the evaluation of patients with lung cancer. 
Cambridge University Press

978-0-521-87202-7 - Lung Cancer

Edited by Sujal R. Desai

Excerpt

More information

\section{Symptoms and Signs due to Para-neoplastic Syndromes}

Para-neoplastic syndromes are present in $10-20 \%$ of patients with lung cancers. Some of the typical syndromes are displayed in Table 1.3 and are usually due to the ectopic production of hormones or peptides. These patients can present with vague symptoms such as tiredness, nausea, abdominal pain or confusion, or more specific symptoms such as galactorrhoea. Ectopic hormone production is more common in small cell lung cancer and some of the cells show neuro-endocrine characteristics.

Table 1.3. Paraneoplastic syndromes

\begin{tabular}{|c|c|c|}
\hline \multicolumn{2}{|l|}{ Common } & \multirow[t]{2}{*}{ Rare } \\
\hline General & General & \\
\hline Anorexia, & Fever & Hypercalcitoninemia \\
\hline Cachexia & Marantic endocarditis & Hypoglycemia \\
\hline Weight loss & & Hypophosphatemia \\
\hline Clubbing of finger nails & Connective Tissue/vasculitis & Lactic acidosis \\
\hline \multirow[t]{2}{*}{ HPOA } & Dermatomyositis/Polymyositis & \\
\hline & Systemic Lupus Erythematosus & Haematological \\
\hline Endocrine & & Amyloidosis \\
\hline Hypercalcemia & Cutaneous & Eosinophilia \\
\hline \multirow[t]{2}{*}{ SIADH } & Acanthosis nigricans & Leucocytosis \\
\hline & Acquired ichthyosis & Leukoerythroblastic reaction \\
\hline Haematological & Acquired palmoplantar keratoderma & Polycythemia \\
\hline Anaemia & Dermatomyositis & Thrombocytopenia \\
\hline \multirow[t]{2}{*}{ Polycythemia } & Erythema annulare & \\
\hline & Exfoliative dermatitis & Neurological \\
\hline Neurological & Pemphigus & Autonomic neuropathy \\
\hline Lambert-Eaton & Pruritis & Cerebellar degeneration \\
\hline myasthenia syndrome & & Limbic encephalitis \\
\hline \multirow[t]{6}{*}{ Peripheral neuropathy } & Endocrine & Pontine myelinosis \\
\hline & Acromegaly & Retinopathy \\
\hline & Carcinoid Syndrome & \\
\hline & Cushings Syndrome & Renal \\
\hline & Gynaecomastia & Glomeronephritis \\
\hline & & Tubulointerstitial \\
\hline
\end{tabular}

Key:

HPOA is hypertrophic pulmonary osteo-arthropathy.

SIADH is syndrome of inappropriate antidiuretic hormone secretion. 
The range of peptides secreted includes adrenocorticotrophic hormone (ACTH), antidiuretic hormone $(\mathrm{ADH})$, calcitonin, oxytocin and parathyroid hormone. Although elevated levels of these peptides are found in patients with lung cancer only about $5 \%$ of patients develop the clinical syndromes. Digital clubbing with hypertrophic pulmonary osteo-arthropathy (HPOA) is considered a nonmetastatic manifestation of lung cancer. Peripheral neuropathy and neurological syndromes such as Lambert-Eaton myasthenic syndrome may also be associated with lung cancer.

\section{Diagnosis of Lung Cancer}

Histopathological and cytological confirmation of the diagnosis is an essential step in the management of patients with lung cancer. Diagnosis and staging can be approached together to provide better prognostic information and more appropriately planned treatment. A computed tomography (CT) scan of the thorax and abdomen should be the initial diagnostic test after chest radiography (Fig. 1.1). The CT study will not only provide information about the location of the primary lesion but also about possible involvement of adjacent structures and provisional staging information (note that the issue of the imaging diagnosis and staging of lung cancer are the subjects of separate chapters in this volume). In addition to CT scanning, many patients with suspected lung cancer will be referred for fibreoptic bronchoscopy. This is discussed in the following section.

\section{Fibroptic Bronchoscopy}

Bronchoscopy is one of the key investigations in the assessment of patients with suspected lung cancer since the procedure not only permits visual examination of the major airways down to subsegmental level, but also provides a variety of methods of sampling abnormal tissue for cytological or histopathological diagnosis. Central tumours vary in appearance from extrinsic polypoid lesions through diffuse plaque-like infiltrations to subtle mucosal irregularity (Fig. 1.2). Large tumour masses and enlarged lymph nodes may also cause extrinsic narrowing of the airways.

With central lesions, a number of sampling techniques such as bronchial washings, bronchial biopsy and bronchial brushings may be utilized. With washings, 

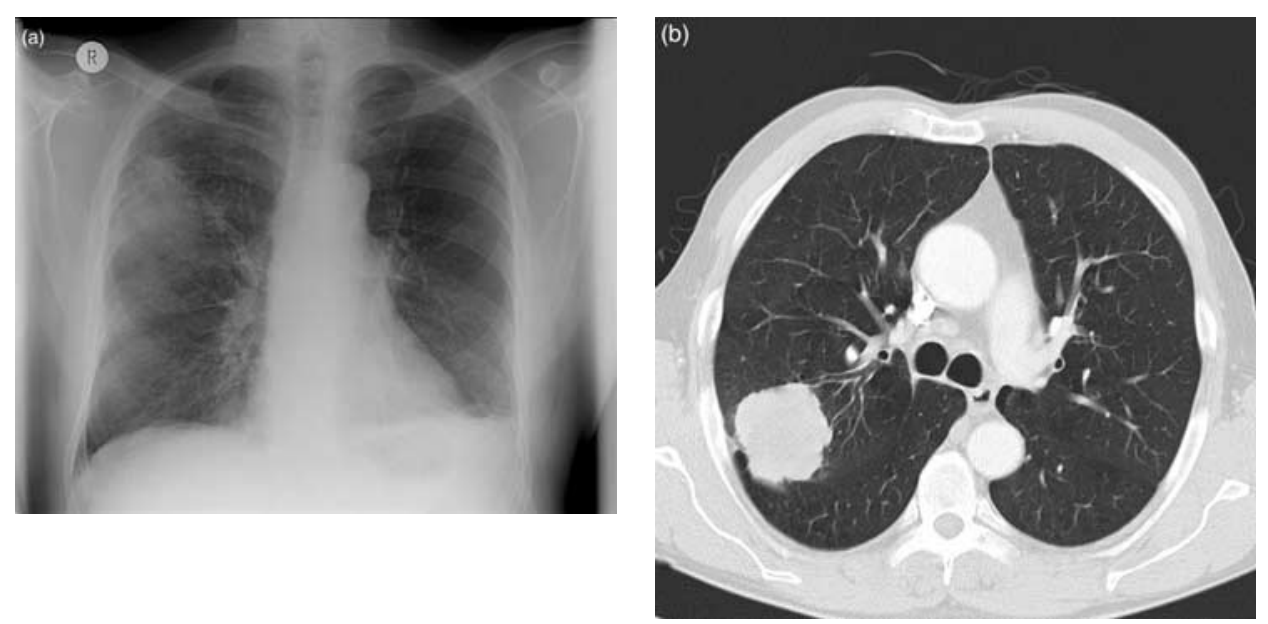

Figure 1.1 (a) Chest radiograph and (b) CT scan demonstrating a right upper lobe mass

approximately $20 \mathrm{ml}$ of saline is instilled around the area of abnormality and the aspirate sent for cytological analysis. Bronchial biopsy is the most useful test for polypoid lesions. Forceps can be inserted through the instrument channel of the bronchoscope to pinch biopsies of the lesion under direct vision. A cytological brush can also be used to scrape some cells from the abnormal area. A combination of these techniques should provide a spot-on diagnosis in up to $90 \%$ of patients where the lesion is located in the larger central airways [12].

Where the lesion is peripheral, techniques such as segmental lavage, selective brushings and fluoroscopic transbronchial fine needle aspiration may be utilized. However, the yield is usually lower at around 40\% [13, 14]. Hence, CT guided biopsy is the usual technique for obtaining a diagnosis in peripherally located lesions. New techniques have been developed which facilitate bronchoscopic sampling of peripheral lesions including magnetic navigation guided bronchoscopy. A spiral CT with $1.5-3 \mathrm{~mm}$ slice thickness is required and specific landmarks marked at virtual bronchoscopy. A catheter with a magnetic tracking device is then inserted through the instrument channel (Super Dimensions Bronchus ${ }^{\mathrm{TM}}$ ) and the catheter tip is positioned at the same landmarks and calibrated with the CT scan. This allows the CT data to be overlayed on the patient and the system can then be used to guide the catheter with the magnetic tracking device to the target lesion. Once the target is reached, the tracking device is removed, the biopsy forceps or 
Cambridge University Press

978-0-521-87202-7 - Lung Cancer

Edited by Sujal R. Desai

Excerpt

More information

8

Pallav Shah
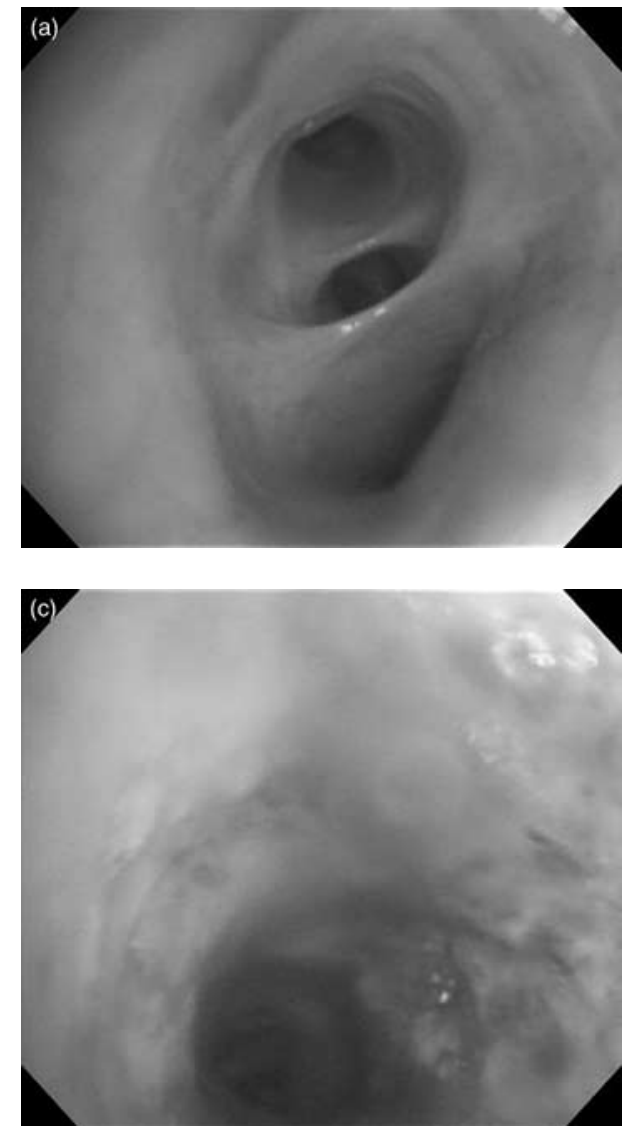

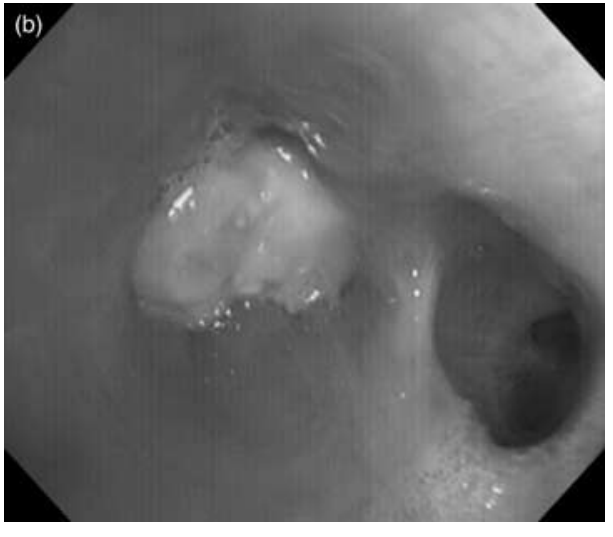

Figure 1.2 Videobronchoscopy appearance of: (a) normal endobronchial airway at segmental level (b) polypoid exophytic tumour; (c) submucosal lesion (for a colour version of this figure please see the colour plate section)

needle is inserted through the catheter and appropriate samples obtained for diagnosis.

Transbronchial fine needle aspiration (TBNA) is a technique that is being incorporated into the routine clinical assessment of patients with suspected lung cancer $[15,16]$. It enables sampling of mediastinal and hilar lymph nodes and provides both diagnostic and staging information. The procedure should be planned according to data provided by a recent CT study. The TBNA needle is inserted through the bronchoscopic channel then inserted at the appropriate point through the airway (Fig. 1.3). The needle is pushed all the way through and suction is applied to the other end with a $20 \mathrm{ml}$ syringe. A jabbing action during the procedure allows cytological material to be aspirated into the needle. The sample obtained is then spread on slides or injected into a liquid media and 
Cambridge University Press

978-0-521-87202-7 - Lung Cancer

Edited by Sujal R. Desai

Excerpt

More information
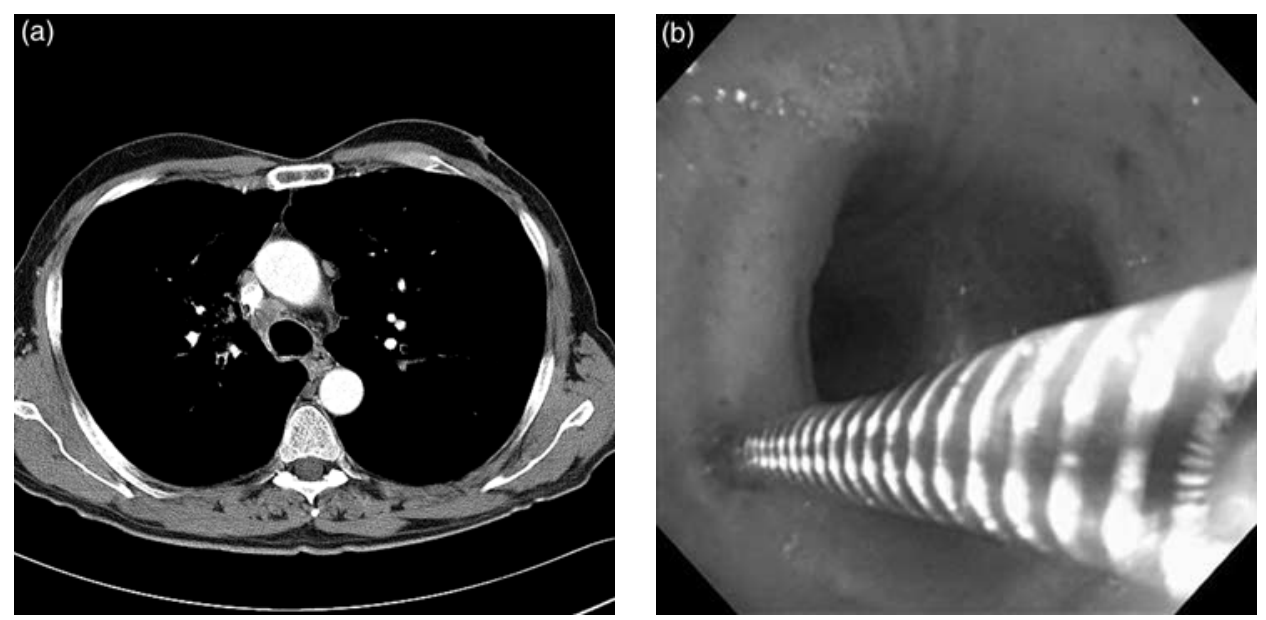

Figure 1.3 (a) CT scan of a patient with right upper lobe tumour and enlarged right paratracheal lymph node and (b) videobronchoscopy appearance of the trachea demonstrating transbronchial fine needle aspiration of the right paratracheal lymph node (for a colour version of this figure please see the colour plate section)

sent for cytological analysis. TBNA should be performed before inspection of the airways so as not to contaminate the samples and minimize the risk of false positive results. This is important as TBNA in this context provides both diagnostic and staging information. On site cytological analysis appears to enhance the diagnostic yield of TBNA and reduces the number of samples that have to be obtained for diagnosis [17]. It must be remembered that TBNA results do not exclude neoplastic disease and should be followed up by further investigations, such as mediastinoscopy, in appropriate cases. TBNA is a safe and effective technique. Complications are rare and consist of pneumothorax, pneumomediastinum and bleeding $[15,16]$.

Puncture bronchoscopes have been developed which integrate video bronchoscopes with a linear array ultrasound probe to improve the diagnostic yield from TBNA. This integrated bronchoscope can be used to assess the mediastinum accurately, which significantly improves the diagnosis and staging of patients with suspected lung cancer. The bronchoscope has a dedicated needle, which can be inserted through the instrument channel of the bronchoscope so TBNA can be performed with real time ultrasound imaging. A preliminary study in 70 patients has demonstrated a high sensitivity (95.7\%) and accuracy (97.1\%) in patients with suspected malignancy [18]. 
Cambridge University Press

978-0-521-87202-7 - Lung Cancer

Edited by Sujal R. Desai

Excerpt

More information

\section{Prognosis}

The three main prognostic factors for bronchogenic carcinoma are cell type, disease staging or extent and treatment modality. Small cell lung cancer carries the worst prognosis and untreated median survival is only around three months. With chemotherapy this improves to around nine months. In contrast, survival of non-small cell lung cancer is better. Disease stage strongly influences treatment and survival. Patients with Stage I disease who are amenable to surgery have a five-year survival rate - over $60 \%$ - whereas patients with stage III or IV disease who are only amenable to palliative treatment have a five-year survival rate of less than $10 \%$. The EUROCARE data demonstrate marked variations in survival between European countries [19]. For example, one-year and five-year survival rates in Finland were $43 \%$ and $16 \%$, respectively, compared to $23 \%$ and $6 \%$ in the United Kingdom. Other prognostic factors are age. The EUROCARE data demonstrate a poorer prognosis with increasing age [19]. This relationship is maintained with cell type and disease stage.

\section{Summary}

Smoking is the main aetiological factor in the pathogenesis of lung cancer. Despite this knowledge, the incidence and mortality for lung cancer remains high. Symptoms occur late in the natural history of lung cancer and hence the majority of lung cancers present late with advanced disease. Accurate diagnosis and staging are an essential guide to treatment and prognosis.

\section{REFERENCES}

1. Quinn, M., Babb, P., Brock, A., Kibly, L., Jones, J. (2001). Cancer Trends in England \& Wales 1950-1999. London: Office for National Statistics.

2. Stat Bite (2005). US death rates for selected cancers in men, 1969-2002. J Natl Cancer Inst., 97(18), 1328.

3. Ferley, J., Bjalk, R. J., Pisani, P., et al. (1996). Eucan90: Cancer in the European Union. IARC Cancer base ND 1. lyon: IARc.

4. Doll, R., Hill, A. B. (1950). Smoking and cancer of the lung; preliminary report. Br Med J, 2, 739.

5. Doll, R., Hill, A.B. (1954). The mortality of doctors in relation to their smoking habits. A preliminary report. $\mathrm{Br}$ Med J, 4877, 1451. 